\title{
Characterizing New Energy Materials Using a Multi-Technique Approach
}

\author{
T. S. Nunney*, R. G. White*, N. Bulloss**, P. Camus**, J. Konopka**, H. M. Meyer III*** \\ * Thermo Fisher Scientific, The Birches, Imberhorne Lane, East Grinstead, West Sussex, RH19 \\ 1UB, UK. \\ ** Thermo Fisher Scientific, 5225 Verona Rd, Madison, WI 53711 \\ *** Materials Science and Technology Division, Oak Ridge National Laboratory, 1 Bethel Valley \\ Road, Oak Ridge, TN, 37831-6064
}

In order to meet the challenges of more economical and environmentally benign energy production, a new generation of complex materials and devices is being developed, such as thin film solar cells, fuel cells, and batteries. In all stages of development there is a requirement for materials characterization and analysis; from the initial development stages, through to testing of the finished article. Most materials need to be analyzed for compositional homogeneity across the sample surface and also for layer chemistry and thickness through the sample. It is rare that a single technique can achieve these testing requirements, and therefore a complementary approach involving several techniques is demanded.

In this presentation we will discuss how a multi-technique approach can address a variety of technical problems, illustrated by examples from real applications case studies. We will mainly concentrate on the information supplied from two techniques, XPS and EDS, but we will also consider the additional data that can be obtained from other sources such as Raman spectroscopy.

X-ray photoelectron spectroscopy (XPS) is ideally suited to the determination of the surface chemistry and the way in which that chemistry changes in the surface and near-surface region. The technique provides quantitative elemental and chemical information with extremely high surface sensitivity and is ideal for comprehensively characterising the elemental composition and chemical bonding states at surfaces and interfaces.

Energy Dispersive Spectroscopy (EDS) is an analytical technique used in electron microscopes to determine sample chemistry. EDS collects characteristic $\mathrm{x}$-rays generated by the rastering scan of the electron beam to generate a full elemental $x$-ray spectrum at each pixel of the electron image. The latest generation EDS silicon drift detectors are capable of collecting and storing hundreds of thousands of x-ray counts per second. This large volume of $x$-ray data collected across the sample allows for rapid identification and characterization of surface defects and lateral compositional variations. Software advances now allow rapid, multivariate statistical analysis processing of very limited amounts of x-ray data to determine not only the elemental distribution across the sample but also the chemical phase distribution.

Figures 1-3 show how the complementary techniques of XPS and EDS have been applied to the investigation of a delaminated CIGS solar device. 


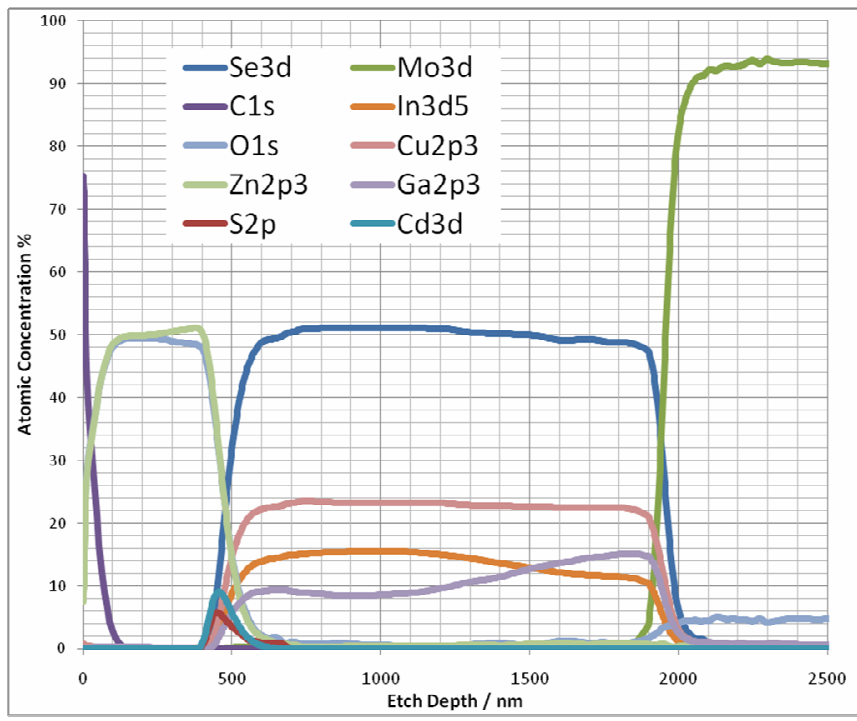

Fig. 1. XPS elemental depth profile of a CIGS solar cell device

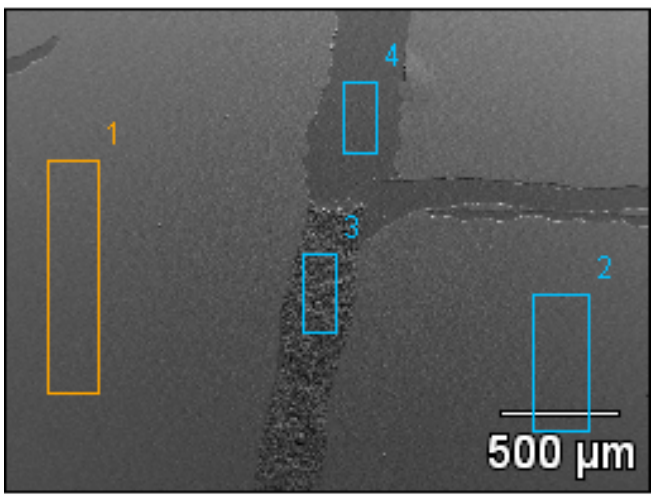

Fig. 2 Areas on a delaminated CIGS device surface selected for EDS analysis

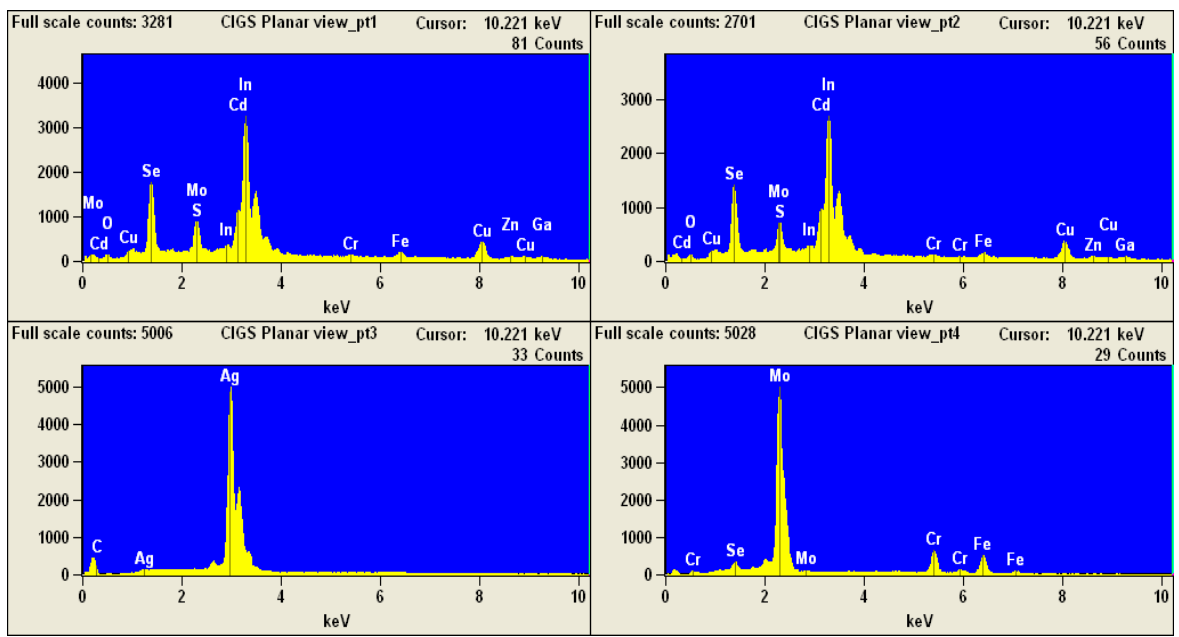

Fig. 3 EDS spectra corresponding to selected locations in Fig. 2. 